BULLETIN OF THE

AMERICAN MATHEMATICAL SOCIETY

Volume 81, Number 2, March 1975

\title{
THE LEFSCHETZ NUMBER AND FIBER PRESERVING MAPS
}

\author{
BY J. C. BECKER, A. CASSON AND D. H. GOTTLIEB \\ Communicated by E. H. Brown, Jr., November 5, 1974
}

1. Introduction. Let $p: E \rightarrow B$ be a Hurewicz fibration whose base $B$ and fiber $F$ are homotopy equivalent to a finite complex, and let $f: E \rightarrow E$ be a fiber preserving map (over the identity). Let $d: \Omega B \rightarrow F$ denote the transgression map which arises from the Puppe sequence of the fibration. Our purpose is to relate the homomorphisms induced by the projection $p$ and the transgression $d$ with the Lefschetz number of $g: F \rightarrow F$, the restriction of $f$ to the fiber.

THEOREM 1. There is an S-map $\tau_{f}: B^{+} \rightarrow E^{+}$such that the composite

$$
H^{*}(B) \stackrel{p^{*}}{\longrightarrow} H^{*}(E) \stackrel{\tau_{f}^{*}}{\longrightarrow} H^{*}(B)
$$

is multiplication by the Lefschetz number $\Lambda_{g}$ of $g$.

Theorem 2. For $k>0, \Lambda_{g} d^{*}=0: H^{k}(F) \rightarrow H^{k}(\Omega B)$.

Here $H$ denotes singular cohomology with arbitrary coefficients.

We call $\tau_{f}$ a transfer map. It is a generalization of the transfer for coverings [6], [7] and for fiber bundles [1], [2], [3], [5]. A. Dold [4] has also defined transfer for a large category of maps.

The existence of the transfer leads to various restrictions on the algebraic invariants attached to a fibration with base and fiber a finite complex. In particular we have

Corollary 1. Let $f: E \rightarrow E$ be a fiber preserving map. Then

(a) $p^{*} \otimes 1: h^{*}(B) \otimes Z\left[\Lambda_{g}^{-1}\right] \rightarrow h^{*}(E) \otimes Z\left[\Lambda_{g}^{-1}\right]$ is a split monomorphism for any cohomology theory $h$.

(b) $\Lambda_{g} \partial_{\#}: \pi_{n}(B) \rightarrow \pi_{n-1}(F)$ is trivial, $n<2$ (connectivity of $F$ ).

We will outline two constructions of the transfer each of interest in its own right. The first involves a reduction of the fibration case to the smooth

AMS (MOS) subject classifications (1970). Primary 55 B20, 55 F05; Secondary 55C20, 57D10.

Key words and phrases. Transfer, fibration, Lefschetz number, smoothing, SpanierWhitehead duality. 
fiber bundle case. The second is a direct construction for fibrations, involving Spanier-Whitehead duality. Details of the results announced here and further applications will appear elsewhere.

2. Smooth fiber bundles. Let $p: E \rightarrow B$ be a smooth fiber bundle with base $B$ and fiber $F$ closed manifolds, and let $f: E \rightarrow E$ be a fiber preserving map. To construct the transfer in this case, choose an embedding $E \rightarrow$ $B \times R^{s}$ homotopic to $p$. Its normal bundle $\beta$ is inverse to the bundle $\alpha$ of tangents along the fiber. Let $c: B^{+} \wedge S^{s} \rightarrow E^{\beta}$ denote the Pontryagin-Thom map, $\Delta: E \rightarrow E^{2}$ the diagonal inclusion into the fiber square and $\pi_{1}: E^{2} \rightarrow$ $E$ projection onto the first factor. Since $\Delta(E)$ has normal bundle $\alpha$ we have $c^{\prime}:\left(E^{2}\right)^{\pi *(\beta)} \rightarrow E^{\alpha \otimes \beta}=E^{+} \wedge S^{s}$. Define $\tau_{f}$ to be

$$
B^{+} \wedge S^{s} \stackrel{c}{\longrightarrow} E^{\beta} \stackrel{(\widetilde{1, f})}{\longrightarrow}\left(E^{2}\right)^{\pi *(\beta)} \stackrel{c^{\prime}}{\longrightarrow} E^{+} \wedge S^{s},
$$

where $(1, f): E \rightarrow E^{2}$ sends $e$ to $(e, f(e))$. Then $\tau_{f}$ meets the requirements of Theorem 1 .

3. Fiber smoothing theorems. Let $F \rightarrow E \rightarrow B$ be a fibration such that $B$ is a closed smooth manifold and $F$ is a finite complex. We have

THEOREM 3 (Open fiber smoothing theorem). There exists an open regular neighborhood $U$ of $F$ and a smooth fiber bundle $U \rightarrow E^{\prime} \rightarrow B$ which is fiber homotopy equivalent to $F \rightarrow E \rightarrow B$.

Let $T^{n}$ denote the $n$-dimensional torus, $n=\operatorname{dim}(B)$.

THEOREM 4 (Closed fiber smoothing theorem). There exists a closed regular neighborhood $W$ of $F$ and a smooth fiber bundle $W \times T^{n} \rightarrow E^{\prime} \rightarrow B$ which is fiber homotopy equivalent to $F \times T^{n} \rightarrow E \times T^{n} \rightarrow B$.

The extension of the transfer from smooth fiber bundles to fibrations is accomplished by the closed fiber smoothing theorem, which asserts that any fibration $p: E \rightarrow B$, as above, is a retract of a smooth fiber bundle $p^{\prime}: E^{\prime} \rightarrow$ $B$. Let $E \stackrel{\lambda}{\longrightarrow} E^{\prime} \stackrel{\rho}{\longrightarrow} E$ denote the asserted inclusion and retraction. If $f: E \rightarrow E$ is fiber preserving, $\tau_{f}$ is then defined to be $\rho \tau_{\lambda f \rho}$.

The general case, i.e. where $B$ is a finite complex can be reduced to the above case by embedding $B$ as a retract of a closed smooth manifold.

4. Duality. A direct construction of transfer for fibrations is based on the following observation. Let $\mu: S^{s} \rightarrow \hat{F} \wedge F^{+}$be a duality map. Then $\hat{F} \wedge F^{+}$is self dual in a canonical way, so that $\mu$ has a $2 s$-dual $\hat{\mu}: \hat{F} \wedge F^{+} \rightarrow S^{s}$. 
LemmA. Let $g: F \longrightarrow F$. The composite $S^{s} \stackrel{\mu}{\longrightarrow} \hat{F} \wedge F^{+} \stackrel{1 \wedge g^{+}}{\longrightarrow} \hat{F} \wedge$ $F^{+} \stackrel{\hat{\mu}}{\longrightarrow} S^{s}$ has degree $\Lambda_{g}$.

By an ex-fibration we mean an object $E=(E, B, p \Delta)$ where $p: E \rightarrow B$ is a fibration, $\Delta$ is a cross section, and (a) $p$ has a lifting function with the property that if $\sigma$ is a path in $B$ its lifting in $E$ which begins at $\Delta(\sigma(0))$ is $\Delta \sigma$. (b) $E \times\{0\} \cup \Delta(B) \times I$ is a vertical retract of $E \times I$. If $p: E \rightarrow B$ is a fibration we have an ex-fibration $\vec{E}$. The disjoint union of $E$ and $B$, with $p$ : $E \rightarrow B$ and $\bar{\Delta}: B \rightarrow \bar{E}$ the obvious maps.

THEOREM 5. If $E$ is an ex-fibration there exists for some integer s, an ex-fibration $E$ and an ex-map $\mu: S^{s} \times B \rightarrow \hat{E} \wedge_{B} \bar{E}$ such that the restriction of $\mu$ to each fiber is a duality map.

Once the existence of dual ex-fibrations is established the remaining aspects of Spanier-Whitehead duality carry over easily to ex-fibrations over a fixed space $B$. To define transfer for a fibration $p: E \rightarrow B$ and fiber preserving map $f: E \rightarrow E$ let $\hat{E}$ be dual to $\bar{E}$. We have

$$
S^{s} \times B \stackrel{\mu}{\longrightarrow} \hat{E} \wedge_{B} \bar{E} \stackrel{1 \wedge(f, 1)}{\longrightarrow} \hat{E} \wedge_{B} \bar{E} \stackrel{\hat{\mu} \wedge 1}{\longrightarrow} S^{s} \wedge_{B} \bar{E}
$$

Identifying the section to a point on each side yields $\tau_{f}: S^{s} \wedge B^{+} \rightarrow S^{s} \wedge$ $E^{+}$. It follows from the Lemma that $\tau_{f}$ meets the requirements of Theorem 1 .

\section{REFERENCES}

1. J. C. Becker, Characteristic classes and $K$-theory, Proceedings of the Binghampton New York Conference on Topology (to appear).

2. J. C. Becker and D. H. Gottlieb, The transfer map and fiber bundles, Topology 211 (1974), 277-288.

Ann. (to appear).

3. Applications of the evaluation map and transfer map theorems, Math.

4. A. Dold, Transfert des points fixes d'une famille continue d'applications, C. R. Acad. Sci. Paris (to appear).

5. D. H. Gottlieb, Fiber bundles and the Euler characteristic, J. Differential Topology (to appear).

6. D. S. Kahn and S. B. Priddy, Applications of the transfer to stable homotopy theory, Bull. Amer. Math. Soc. 78 (1972), 981-987. MR 46 \#8220.

7. F. W. Roush, Transfer in generalized conomology theories, Thesis, Princeton University, Princeton, N. J., 1971.

DEPARTMENT OF MATHEMATICS, PURDUE UNIVERSITY, WEST LAFAYETTE, INDIANA 47907

DEPARTMENT OF MATHEMATICS, CAMBRIDGE UNIVERSITY, CAMBRIDGE, 